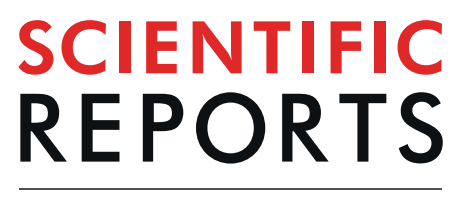

natureresearch

\title{
OPEN Protective Effect of Statins on Pulmonary Hypertension in Chronic Obstructive Pulmonary Disease Patients: A Nationwide Retrospective, Matched Cohort Study
}

\begin{abstract}
Wen-Ting W $\mathbf{u}^{1,2}$ \& Chung-Yu Chen ${ }^{1,3,4^{*}}$
In Taiwan, patients with pulmonary hypertension $(\mathrm{PH})$ related to chronic obstructive pulmonary disease (COPD) are most common PH population (group 3). However, efficacy of medical treatments and optimal prevention methods in this group remain uncertain. Statins such as indirect RhoA/Rho-kinase inhibitors influence one of key signalling pathways that promote PH onset. In this study, we explored protective effects of statins against PH in COPD patients using database from Taiwan National Health Insurance programme from 2002 to 2017. The main outcome was the risk of PH. The Cox proportionalhazards model and the Fine and Gray model were used to adjust covariate and competing risks to estimate the subdistribution hazard ratios (sHRs). 553,617 newly diagnosed COPD patients were stratified by statin users $(n=41,168)$ and statin nonusers $(n=512,449)$. After $1: 1$ propensity score matching of statin users $(n=41,163)$, and 41,163 statin nonusers were included for outcome analysis. Statin users had a $22 \%$ lower risk of PH than nonusers (sHR: $0.78,95 \%$ confidence interval: $0.65-0.94$ ). During subgroup analysis, taking higher daily doses and for a longer duration displayed a more significantly reduced risk of $\mathrm{PH}$ (both $\mathrm{P}$ for trend $<0.001$ ). Statins may have a protective effect against $\mathrm{PH}$ that is dose- and time-dependent.
\end{abstract}

Pulmonary hypertension (PH) is a pathophysiologic and hemodynamic condition that increases the pressure level in the pulmonary arteries, veins and/or capillaries. Eventually, under these conditions, the need for the heart ventricles to contract more and more to pump blood through the lungs and heart can result in heart failure, cardiovascular diseases, or respiratory function loss $^{1}$. In 2018, the sixth World Symposium on PH (WSPH) released an updated statement separating $\mathrm{PH}$ patients into five groups according to clinical presentation, hemodynamic characteristics, pathophysiology, and therapeutic strategy ${ }^{2}$. The incidence of $\mathrm{PH}$ is about 2.4 cases per million adults per year in the United States ${ }^{3-6}$. One Taiwan prevalence survey suggested the patients with PH related to COPD (group 3 in the WSPH classification scheme) is the most common PH population in Taiwan ${ }^{7}$. Of interest, while the severity of $\mathrm{PH}$ among patients in group 3 may be less than that in other PH groups, the three-year survival rate in this group is the lowest across all five $\mathrm{PH}$ groups ${ }^{8}$. This lower survival rate might due to a lack of evidence and efficacy regarding the use of current medical treatments for $\mathrm{PH}$ including supportive therapy and certain drugs in $\mathrm{PH}$ related to COPD. Besides, there has been no clear prevention strategy revealed that can reduce the risk of $\mathrm{PH}$ in COPD patients; currently, only long-term oxygen therapy can improve symptoms. Given these facts, the development of a new treatment in this population is necessary $y^{9,10}$.

\footnotetext{
${ }^{1}$ Master Program in Clinical Pharmacy, School of Pharmacy, Kaohsiung Medical University, Kaohsiung, Taiwan. ${ }^{2}$ Department of Pharmacy, Wan Fang Hospital, Taipei Medical University, Taipei, Taiwan. ${ }^{3}$ Department of Pharmacy, Kaohsiung Medical University Hospital, Kaohsiung, Taiwan. ${ }^{4}$ Department of Medical Research, Kaohsiung Medical University Hospital, Kaohsiung, Taiwan. *email: jk2975525@hotmail.com
} 
There are many studies that have explored the repurposing of $\mathrm{PH}$ treatment drugs on the market for other indications. For example, 3-hydroxy-3-methyl-glutaryl-coenzyme A reductase inhibitors are cholesterol-lowering drugs able to reduce the low-density lipoprotein cholesterol level by around $10 \%$ to $50 \%$ as well as the triglyceride concentration to a small degree. Because of their good lipid-lowering effects, statins are the first-choice therapeutic modalities in hyperlipidemia patients ${ }^{11}$. In addition to their lipid-lowering effects, statins also have displayed anti-inflammatory ${ }^{12-14}$, anti-proliferative $\mathrm{e}^{15-17}$ and anti-thrombotic ${ }^{18,19}$ properties. These effects are associated with the pathophysiology of PH related to COPD. Moreover, statins are indirect RhoA/Rho-kinase inhibitors. Upon blocking the RhoA/Rho-kinase signalling pathway, these medications are capable of stopping vasoconstriction, endothelial nitric oxide synthase, cellular proliferation and apoptosis. However, to date, only in vitro studies have indicated that statins inhibit systemic inflammatory and pulmonary vascular proliferation, and block the RhoA/ Rho-kinase signalling pathway; the efficacy of statins in human clinical trials remains unclear.

As such, we conducted a nationwide, population-based retrospective cohort study to explore whether the protective effects of statins could reduce the risk of $\mathrm{PH}$ in patients with COPD. Moreover, we compared the protective effects of different types of statins and examined whether such effects were dose- or time-dependent.

\section{Results}

Study population. Based on the inclusion and exclusion criteria, a total of 553,617 patients were included in the newly diagnosed COPD cohort (Fig. 1). According to statin exposure, there were s 41,168 statin users and 512,449 nonusers of statins in study population. After 1:1 propensity score (PS) matching, we included 41,163 statin users and 41,163 statin nonusers for $\mathrm{PH}$ outcome analysis. Before PH matching, the mean age of patients in the user group ( 64.60 years) was slightly higher than that in the nonuser group ( 63.95 years). Because of the indications of statins, patients with statins displayed significantly higher rates of dyslipidemia $(\mathrm{P}<0.001)$, coronary artery disease $(\mathrm{P}<0.001)$, and ischaemic stroke $(\mathrm{P}<0.001)$. Most of the comorbidities were found in significantly higher degrees in the user group than in the nonuser group, with the exception of interstitial pulmonary diseases, asthma and malignant and haemorrhagic stroke. In particular, rates of interstitial pulmonary diseases and malignant stroke were similar between the two groups $(\mathrm{P}=0.543$ and $\mathrm{P}=0.250)$. Comedication use presented the same trend of comorbidity, while rates of severe and moderate exacerbations of COPD displayed a significant difference $(\mathrm{P}<0.001)$ between the two user groups, although the majority of patients showed no exacerbation in their condition within one year after the index date. After PS matching, there was a significant difference noted in the distribution of comorbidities and concurrent medication use between the two groups. A Cox proportional-hazards $(\mathrm{CPH})$ model was established to adjust all imbalanced characteristics in the following analysis. The details of baseline characteristics of the COPD cohort are presented in Table 1.

Incidence. A total of $242(0.59 \%)$ users and 338 (0.82\%) nonusers of statins, respectively, experienced $\mathrm{PH}$. The mean follow-up times in the user and nonuser groups were 4.13 and 4.28 years. The statin user group displayed a lower incidence rate of $\mathrm{PH}$ onset as compared with the nonuser group (1.43 vs. 1.97 per 1,000 person-years). The $\mathrm{CPH}$ model analysis findings are summarised in Table 2. In both a univariate analysis [crude hazard ratio (HR): 0.72, 95\% confidence interval (CI): 0.61-0.85; P < 0.001) and multivariate analysis (adjusted HR (aHR): 0.76, 95\% CI: 0.63-0.93; P =0.006), a significantly lower $\mathrm{HR}$ of $\mathrm{PH}$ incidence between statin users and nonusers was observe. There were 6,997 (16.99\%) statin users and 5,553 (13.49\%) statin nonusers who died within the five-year study period. Death may be a competing risk for a $\mathrm{PH}$ event. To adjust the competing risk, we conducted a multivariate with competing risk analysis, where the incidence of $\mathrm{PH}$ still presented a significantly lower subdistribution HR (sHR) between statin users and nonusers (sHR: 0.78, 95\% CI: 0.65-0.94; P=0.010) The above results suggest that statins could reduce the risk of $\mathrm{PH}$ by approximately $28 \%$ to $22 \%$ and provide a protective effect against $\mathrm{PH}$ (Table 2).

Statin users of an older age; male gender; with lower insurance premiums; who were living in the suburbs; and/or who had a cardiac arrhythmia, asthma or heart failure presented a significantly higher risk of developing $\mathrm{PH}$ as compared with nonusers. In contrast, patients with ischaemic stroke or transient ischaemic attack had a lower risk of PH (aHR; 0.75, 95\% CI: 0.59-0.97; P = 0.026) Regarding the COPD severity in sHR analysis, only moderate exacerbation and one-time severe exacerbation significantly increased the risk of PH. We next conducted a stepwise multiple regression analysis to elucidate the main factors affecting the incidence rate of $\mathrm{PH}$. Here, age group, gender, insurance premium, arrhythmia, asthma, heart failure, digoxin, diuretic and COPD severity displayed a significantly different risk. These significant factors were subsequently used to construct an adjusted model for use in the following subgroup analysis (Table 2).

Subgroup analysis. The most used statin was atorvastatin, with nearly $40 \%$ of statin users taking this medication. As indicated in Table 3, most of the statin used showed a trend of a low risk of PH except lovastatin (aHR: 1.63, 95\% CI: 0.95-2.79; $\mathrm{P}=0.076$ ) Among all of those being used, pravastatin had the lowest aHR and appeared to significantly reduce the risk of PH by 56\% (aHR: 0.44, 95\% CI: $0.23-0.86 ; \mathrm{P}=0.016$ ). After adjusting for all-cause mortality as a competing risk, the trend of the protective effect of each statin did not change.

A classification scheme for cumulative defined daily dose (cDDD) including seven levels was included in a multivariate $\mathrm{CPH}$ model analysis. The statin nonuser group was employed as a reference group in this analysis. Table 4 revealed that patients with higher cDDDs had lower aHR values of from 1.36 to 0.26 ( $\mathrm{P}$ for trend $\leq 0.001$ ). Further, among those patients using more than $180 \mathrm{cDDD}$, a significantly lower risk of PH was observed (aHR: 0.58, 95\% CI: $0.42-0.81$; sHR: $0.66,95 \%$ CI: $0.47-0.92$ ). The duration of statin use was calculated by year and divided into six categories. Patients with longer durations of statin use had a lower risk of PH (aHR: 1.15-0.31; P for trend $\leq 0.001$ ), with a significantly lower risk of PH observed among those using statins for more than one year (aHR: 0.44, 95\% CI: 0.31-0.64; sHR: 0.47, 95\% CI: 0.32-0.67). Separately, we divided the frequency of use of statins into four levels and conducted a multivariate $\mathrm{CPH}$ model analysis. The most common frequency of statin use is 30 defined daily doses 


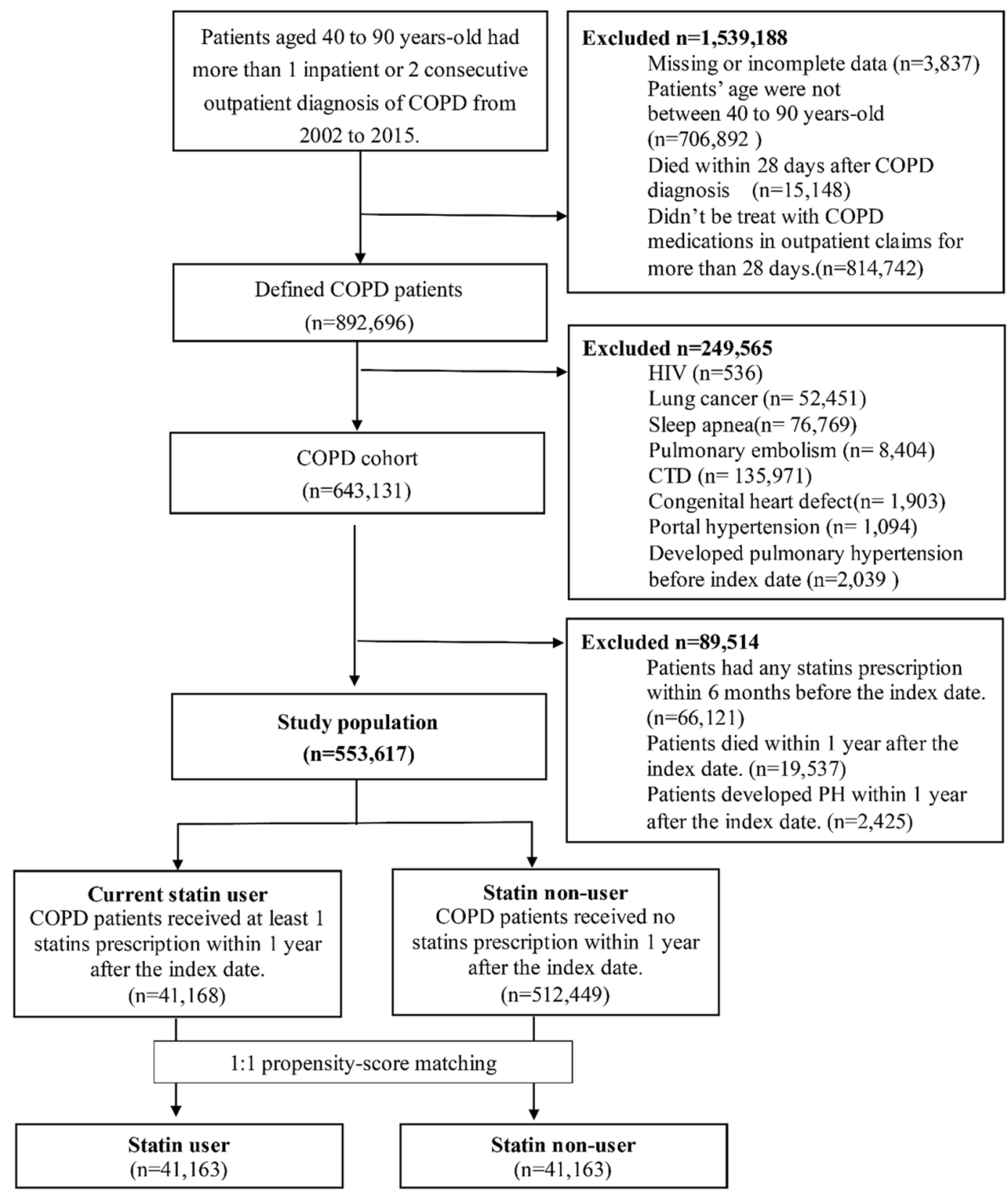

Figure 1. Result of flow chart in study population.

(DDDs) per month. Patients using greater numbers of DDDs of statins per month had a lower risk of PH (aHR: 0.81-0.51; P for trend $\leq 0.001)$. Further, patients who used more than 20 DDDs of statin per month started to show a significantly lower risk of PH (aHR: 0.51, 95\% CI: 0.39-0.67; sHR: 0.54, 95\% CI: 0.41-0.71).

Sensitivity analysis. As compared with the original definition of a PH event, a more precise definition did not change the trend of observing a protective effect against $\mathrm{PH}$. Moreover, the sensitivity analysis had a lower risk of $\mathrm{PH}$ when compared with the original definition of a $\mathrm{PH}$ event in the statin user group (aHR: 0.70, 95\% CI: 0.56-0.87 vs. aHR: $0.76,95 \%$ CI: 0.63-0.93) Besides, extending the one-year confirmation period to three years and conducting a longer or shorter duration of observation did not have much of an influence on the outcome of $\mathrm{PH}$ risk. Findings of the sensitivity analysis are shown in Supplementary Tables 1 and 2.

\section{Discussion}

During the five-year study observation period, the statin user group displayed a lower incidence rate of $\mathrm{PH}$ as compared with in the nonuser group ( 1.43 vs. 1.97 per 1,000 person-years; $\mathrm{P}<0.001$ ). After adjusting for age, sex, income, comorbidity, comedication use, COPD severity and competing risks, the finding was that statin use reduced the incidence rate of $\mathrm{PH}$ by $22 \%$ among COPD patients (sHR: $0.78,95 \%$ CI: $0.65-0.94 ; \mathrm{P}=0.010$ ). These results support the suggestion that statins offer a protective effect against $\mathrm{PH}$ in COPD patients. 


\begin{tabular}{|c|c|c|c|c|c|c|}
\hline \multirow[b]{2}{*}{ Characteristics n (\%) } & \multicolumn{3}{|l|}{ Before matching } & \multicolumn{3}{|l|}{ After matching } \\
\hline & User $\mathrm{N}=41,168$ & $\begin{array}{l}\text { Non-user } \\
N=512,449\end{array}$ & $p$-value & User $N=41,163$ & $\begin{array}{l}\text { Non-user } \\
\mathrm{N}=41,163\end{array}$ & $p$-value \\
\hline Propensity score (SD) & $0.88(0.08)$ & $0.93(0.05)$ & $<0.001$ & $0.75(0.03)$ & $0.75(0.03)$ & 1.000 \\
\hline \multicolumn{7}{|l|}{ Age group } \\
\hline Mean (SD) & $64.60(11.06)$ & $63.95(12.85)$ & $<0.001$ & $64.60(11.06)$ & $64.67(11.27)$ & 0.370 \\
\hline $40 \leq$ age $<50$ & $3,937(9.56)$ & $83,280(16.25)$ & $<0.001$ & $3,936(9.56)$ & $3,936(9.56)$ & 1.000 \\
\hline $50 \leq$ age $<60$ & $10,204(24.79)$ & $117,885(23.00)$ & & $10,204(24.79)$ & $10,204(24.79)$ & \\
\hline $60 \leq$ age $<70$ & $12,356(30.01)$ & $119,985(23.41)$ & & $12,354(30.01)$ & $12,354(30.01)$ & \\
\hline $70 \leq$ age $<80$ & $10,827(26.30)$ & $123,860(24.17)$ & & $10,825(26.30)$ & $10,825(26.30)$ & \\
\hline $80 \leq$ age & $3,844(9.34)$ & $67,439(13.16)$ & & $3,844(9.34)$ & $3,844(9.34)$ & \\
\hline Male & $23,303(56.60)$ & $313,271(61.13)$ & $<0.001$ & $23,301(56.61)$ & $23,301(56.61)$ & 1.000 \\
\hline \multicolumn{7}{|c|}{ Insurance premium (TWD) } \\
\hline$\leq 22,800$ TWD & $21,581(52.42)$ & $280,896(54.81)$ & $<0.001$ & $21,581(52.43)$ & $21,582(52.43)$ & 0.994 \\
\hline$>22,800$ TWD & $19,587(47.58)$ & $231,553(45.19)$ & & $19,582(47.57)$ & $19,581(47.57)$ & \\
\hline \multicolumn{7}{|l|}{ Urbanization level } \\
\hline Urban & $20,416(49.59)$ & $252,309(49.24)$ & 0.201 & $20,411(49.59)$ & $20,346(49.43)$ & 0.646 \\
\hline Suburban & $16,255(39.48)$ & $202,886(39.59)$ & & $16,255(39.49)$ & $16,237(39.45)$ & \\
\hline Rural & $4,497(10.92)$ & $57,254(11.17)$ & & $4,497(10.92)$ & $4,580(10.13)$ & \\
\hline \multicolumn{7}{|l|}{ Comorbidity } \\
\hline Dyslipidemia & $25,510(61.97)$ & $34,381(6.71)$ & $<0.001$ & $25,510(61.97)$ & $4,603(61.18)$ & $<0.001$ \\
\hline Hypertension & $27,917(67.81)$ & $216,905(42.33)$ & $<0.001$ & $27,912(67.81)$ & $27,916(67.82)$ & 0.976 \\
\hline Diabetes Mellitus & $15,840(38.48)$ & $75,574(14.75)$ & $<0.001$ & $15,835(38.47)$ & $16,043(38.97)$ & 0.137 \\
\hline Obesity & $215(0.52)$ & $1,017(0.20)$ & $<0.001$ & $215(0.52)$ & $142(0.34)$ & $<0.001$ \\
\hline Chronic kidney disease & $2,010(4.88)$ & $12,770(2.49)$ & $<0.001$ & $2,010(4.88)$ & $1,668(4.05)$ & $<0.001$ \\
\hline Chronic liver disease & $4,892(11.88)$ & $49,970(9.75)$ & $<0.001$ & $4,892(11.88)$ & $4,820(11.71)$ & 0.437 \\
\hline Arrhythmia & $4,810(11.68)$ & $41,636(8.12)$ & $<0.001$ & $4,805(11.67)$ & $4,739(11.51)$ & 0.472 \\
\hline $\begin{array}{l}\text { Interstitial pulmonary } \\
\text { diseases }\end{array}$ & $177(0.43)$ & $2,310(0.45)$ & 0.543 & $177(0.43)$ & $161(0.39)$ & 0.383 \\
\hline Asthma & $15,607(37.91)$ & $197,980(38.63)$ & 0.004 & $15,604(37.91)$ & $15,760(37.29)$ & 0.263 \\
\hline Malignant & $4,236(10.29)$ & $53,653(10.47)$ & 0.250 & $4,235(10.29)$ & $4,369(10.61)$ & 0.127 \\
\hline \multicolumn{7}{|l|}{ ASCVD } \\
\hline Coronary artery disease & $13,622(33.09)$ & $76,755(14.98)$ & $<0.001$ & $13,618(33.08)$ & $8,400(33.41)$ & $<0.001$ \\
\hline Peripheral vascular disease & $1,582(3.84)$ & $11,217(2.19)$ & $<0.001$ & $1,582(3.84)$ & $1,260(3.06)$ & $<0.001$ \\
\hline Ischemic stroke/TIA & 6,239(15.15) & $45,500(8.88)$ & $<0.001$ & $6,238(15.15)$ & $4,667(15.34)$ & $<0.001$ \\
\hline Hemorrhagic stroke & $583(1.42)$ & $8,447(1.65)$ & $<0.001$ & $583(1.42)$ & $825(1.20)$ & $<0.001$ \\
\hline Heart failure & $6,155(14.95)$ & $42,883(8.37)$ & $<0.001$ & $6,150(14.94)$ & $6,152(14.95)$ & 0.984 \\
\hline $\begin{array}{l}\text { Left ventricular } \\
\text { hypertrophy }\end{array}$ & $435(1.06)$ & $3,154(0.62)$ & $<0.001$ & $435(1.06)$ & $430(1.04)$ & 0.864 \\
\hline \multicolumn{7}{|l|}{ Co-medication } \\
\hline Digoxin & $1,902(4.62)$ & $17,049(3.33)$ & $<0.001$ & $1,898(4.61)$ & $2,045(4.97)$ & 0.016 \\
\hline Oral anticoagulant agents & $1,004(2.44)$ & $6,669(1.30)$ & $<0.001$ & $1,003(2.44)$ & $790(2.92)$ & $<0.001$ \\
\hline Oral antiplatelet agents & $20,260(49.21)$ & $115,688(22.58)$ & $<0.001$ & $20,257(49.21)$ & $13,406(49.57)$ & $<0.001$ \\
\hline Diuretics & $12,105(29.40)$ & $90,184(17.60)$ & $<0.001$ & $12,100(29.40)$ & $10,509(29.53)$ & $<0.001$ \\
\hline СCB & $21,022(51.06)$ & $162,839(31.78)$ & $<0.001$ & $21,019(51.06)$ & $19,511(51.40)$ & $<0.001$ \\
\hline ACEI/ACB & $21,653(52.60)$ & $134,311(26.21)$ & $<0.001$ & $21,648(52.59)$ & $17,584(52.72)$ & $<0.001$ \\
\hline Beta blocker & $14,761(35.86)$ & $97,769(19.08)$ & $<0.001$ & $14,757(35.85)$ & $11,917(35.95)$ & $<0.001$ \\
\hline Metformin & $11,085(26.93)$ & $44,553(8.69)$ & $<0.001$ & $11,080(26.92)$ & $9,567(26.24)$ & $<0.001$ \\
\hline Fibrate & $4,875(11.84)$ & $14,045(2.74)$ & $<0.001$ & $4,873(11.84)$ & $2,080(11.05)$ & $<0.001$ \\
\hline Other lipid-lowering drugs & $186(0.45)$ & $541(0.11)$ & $<0.001$ & $186(0.45)$ & $64(0.16)$ & $<0.001$ \\
\hline \multicolumn{7}{|l|}{ Medication for COPD } \\
\hline LABA & $933(2.27)$ & $11,143(2.17)$ & 0.220 & $933(2.27)$ & $859(2.09)$ & 0.077 \\
\hline LABA/ICS & $4,126(10.02)$ & $48,234(9.41)$ & $<0.001$ & $4,125(10.02)$ & $3,515(10.54)$ & $<0.001$ \\
\hline LAMA & $1,614(3.92)$ & $17,393(3.39)$ & $<0.001$ & $1,614(3.92)$ & $1,187(3.88)$ & $<0.001$ \\
\hline LABA/LAMA & $214(0.52)$ & $1,814(0.35)$ & $<0.001$ & $214(0.52)$ & $132(0.32)$ & $<0.001$ \\
\hline SABA & $3,261(7.92)$ & $42,497(8.29)$ & 0.008 & $3,260(7.92)$ & $3,244(7.88)$ & 0.836 \\
\hline SAMA & $1,138(2.76)$ & $17,985(3.51)$ & $<0.001$ & $1,137(2.76)$ & $1,447(2.52)$ & $<0.001$ \\
\hline SABA/SAMA & $1,588(3.86)$ & $19,001(3.71)$ & 0.123 & $1,588(3.86)$ & $1,407(3.42)$ & 0.001 \\
\hline Systemic beta- 2 agonists & $10,621(25.80)$ & $129,995(25.37)$ & 0.052 & $10,618(25.80)$ & $10,859(25.38)$ & 0.056 \\
\hline
\end{tabular}




\begin{tabular}{|c|c|c|c|c|c|c|}
\hline \multirow[b]{2}{*}{ Characteristics n (\%) } & \multicolumn{3}{|l|}{ Before matching } & \multicolumn{3}{|l|}{ After matching } \\
\hline & User $\mathrm{N}=41,168$ & $\begin{array}{l}\text { Non-user } \\
\mathrm{N}=512,449\end{array}$ & $p$-value & User $\mathrm{N}=41,163$ & $\begin{array}{l}\text { Non-user } \\
\mathrm{N}=41,163\end{array}$ & $p$-value \\
\hline ICS & $1,190(2.89)$ & $15,262(2.98)$ & 0.314 & $1,189(2.89)$ & $1,102(2.68)$ & 0.065 \\
\hline Methylxanthines & $22,245(54.03)$ & $253,261(49.42)$ & $<0.001$ & $22,241(54.03)$ & $21,248(54.62)$ & $<0.001$ \\
\hline \multicolumn{7}{|l|}{ COPD severity } \\
\hline \multicolumn{7}{|c|}{ Moderate exacerbations } \\
\hline 0 & $36,309(88.20)$ & $446,596(87.15)$ & $<0.001$ & $30,446(73.96)$ & $30,522(73.15)$ & 0.799 \\
\hline 1 & $3,385(8.22)$ & $41,982(8.19)$ & & $4,480(10.88)$ & $4,469(10.86)$ & \\
\hline$\geq 2$ & $1,474(3.58)$ & $23,871(4.66)$ & & $6,237(15.15)$ & $6,172(15.99)$ & \\
\hline \multicolumn{7}{|l|}{ Severe exacerbations } \\
\hline 0 & $30,446(73.96)$ & $371,050(72.41)$ & $<0.001$ & $36,304(88.20)$ & $36,307(88.20)$ & 0.995 \\
\hline 1 & $4,485(10.89)$ & $55,841(10.90)$ & & $3,385(8.22)$ & $3,387(8.23)$ & \\
\hline$\geq 2$ & $6,237(15.15)$ & $85,558(16.70)$ & & $1,474(3.58)$ & $1,469(3.57)$ & \\
\hline
\end{tabular}

Table 1. Baseline characteristics of COPD patients before and after1-to-1propensity score matching, stratified according to statins using. $(\mathrm{COPD}=$ chronic obstructive pulmonary disease; $\mathrm{TWD}=$ Taiwan dollars; ASCVD = atherosclerotic cardiovascular disease; TIA = Transient ischemic attack; $\mathrm{CCB}=$ calcium channel blocker; $\mathrm{ACEI}=$ angiotensin converting enzyme inhibitor; $\mathrm{ARB}=$ angiotensin receptor blocker; LABA = Long-acting $\beta_{2}$-aginist; LAMA = Long-acting muscarinic antagonists; SABA = Short-acting $\beta_{2}$-aginist; SAMA $=$ Short-acting muscarinic antagonists; $\mathrm{ICS}=$ Inhaled corticosteroid).

The pathophysiology in PH related to COPD is complex and caused by multiple mechanisms. Pulmonary vascular remodelling, parenchymal lung destruction and hypoxia are the three known main independent mechanisms in PH related to COPD. Among these, pulmonary vascular remodelling and parenchymal lung destruction are observed early on during the course of $\mathrm{PH}$. These conditions may also be associated with findings of systemic inflammation and endothelial cell dysfunction that are involved in the pathophysiology of COPD as well. If COPD continues to deteriorate toward hypoxemia, the onset of hypoxia causes pulmonary arterial vasoconstriction ${ }^{9}$. The protective effect of statins may be attributed to their anti-inflammatory effect that functions by limiting immune cell activation and reducing inflammatory cytokines ${ }^{12-14}$. A controlled pilot study showed that atorvastatin treatment in COPD patients significantly reduced the neutrophil count in sputum by $34 \%$ and the CD $45+$ cell count by $57 \%$ in lung biopsies $(\mathrm{P}=0.008)^{13}$. Moreover, statins are the same as endothelin-1 receptor antagonists, which are specific drugs used for $\mathrm{PH}$ treatment in that endothelin-1 receptor antagonists are indirect RhoA/Rho-kinase inhibitors ${ }^{20}$. RhoA is one of the Rho G proteins, which are intracellular messengers. In the RhoA/Rho-kinase signalling pathway, RhoA activates the downstream effectors Rho-kinase I (ROCK-I) and Rho-kinase II (ROCK-II) and causes vasoconstriction, endothelial nitric oxide synthase, cellular proliferation and apoptosis. The expression of the signalling pathway is common in hypoxic lungs. Statins can decrease the progression of an early process in the cholesterol biosynthetic pathway as well as inhibit the synthesis of isoprenoids, which are prerequisite posttranslational lipid attachments necessary for Rho activation ${ }^{20}$. Statins also have anti-thrombotic effects: they inhibit the platelet-derived growth factor signal and reduce platelet thrombus formation in patients with idiopathic pulmonary arterial hypertension. Fluvastatin has shown beneficial effects on chronic hypoxia-induced PH by limiting endothelial nitric oxide synthase activity ${ }^{18,19}$. In summary, because of their role as indirect RhoA/Rho-kinase inhibitors and other pharmacological activities, we hypothesised that statins may have potential therapeutic benefits in $\mathrm{PH}$.

To our knowledge, this study was the first to investigate whether statins can reduce the incidence of $\mathrm{PH}$ in the COPD population. The only other similar study involving patients with severe COPD supported that statin use is associated with a significantly lower PAWP $(12 \pm 5$ vs. $15 \pm 6 \mathrm{mmHg} ; \mathrm{P}=0.002)$ and PAPm $(26 \pm 7$ vs. $29 \pm 7 \mathrm{mmHg} ; \mathrm{P}=0.002$ ) outcomes ${ }^{21}$. Findings of PAWP values over $15 \mathrm{mmHg}$ and PAPm values over $20 \mathrm{mmHg}$ lead to placement in the WSPH's group 3. So, the reduction of PAWP and PAPm via statin use may support that statins have an association with the reduction of $\mathrm{PH}$ incidence. In this manner, these results complement our study findings. However, the incidence of group $3 \mathrm{PH}$ is still unknown. One study reported an incidence of $14 \%$ among elderly patients older than 65 years, while the ASPIRE registry data showed that $56.7 \%$ of COPD patients had group $3 \mathrm{PH}^{8,22}$. It is difficult to compare the incidence and prevalence rates among these studies and our research because the study populations vary. In our multiple regression analysis, older age, male gender, lower insurance premiums, living in the suburbs, having an arrhythmia, having asthma, having heart failure and COPD severity were 4 risk factors for PH development. According to the pathophysiology and disease progression of $\mathrm{PH}$, these risk factors appear reasonable ${ }^{9}$. However, patients with ischaemic stroke or transient ischaemic attack had a lower risk of PH (aHR: 0.75, 95\% CI: 0.59-0.97; P = 0.026) The reasoning for this perhaps is that patients who experience ischaemic stroke have a ninefold higher mortality rate as compared with patients without stroke: in other words, they die before $\mathrm{PH}$ occurs, leading to a lower reported incidence of $\mathrm{PH}^{23}$. After adjusting the all-cause mortality as a competing risk, statin users still showed a significantly lower risk of PH in comparison with nonusers (sHR: 0.78, 95\% CI: 0.65-0.94; $\mathrm{P}=0.010$ ).

During subgroup analysis, the results indicated dose- and time-dependent effects existed for statin use in that the statins achieved the protective effect against $\mathrm{PH}$ until patients achieved greater than $180 \mathrm{cDDD}$, reached one year of use, or used more than 20 DDDs per month. Among patients with greater cDDDs and longer durations of use, a lower risk of $\mathrm{PH}$ incidence could be observed. To understand the association between cDDD and the 


\begin{tabular}{|c|c|c|c|c|c|c|}
\hline \multirow[b]{2}{*}{ Variables } & \multirow{2}{*}{$\begin{array}{l}\text { Crude } \\
\text { HR }(95 \% \mathrm{CI})\end{array}$} & \multirow[b]{2}{*}{$p$-value } & \multirow{2}{*}{$\begin{array}{l}\text { Adjusted } \\
\text { HR }^{\mathrm{a}}(95 \% \mathrm{CI})\end{array}$} & \multirow[b]{2}{*}{$p$-value } & \multirow{2}{*}{$\begin{array}{l}\text { Subdistribution } \\
\text { HR }^{\mathrm{a}}(95 \% \mathrm{CI}) \\
\end{array}$} & \multirow[b]{2}{*}{$p$-value } \\
\hline & & & & & & \\
\hline User $v s$. non-user & $0.73(0.61-0.86)^{* * *}$ & $<0.001$ & $0.76(0.63-0.93)^{* *}$ & 0.007 & $0.78(0.65-0.94)^{*}$ & 0.010 \\
\hline \multicolumn{7}{|l|}{ Age group $^{b}$} \\
\hline $40 \leq$ age $<50$ & 1 (reference) & & 1 (reference) & & 1 (reference) & \\
\hline $50 \leq$ age $<60$ & $1.55(0.95-2.53)$ & 0.080 & $1.44(0.88-2.35)$ & 0.150 & $1.43(0.87-2.34)$ & 0.154 \\
\hline $60 \leq$ age $<70$ & $2.79(1.76-4.44)^{* * * *}$ & $<0.001$ & $2.05(1.28-3.29)^{* * *}$ & 0.003 & $2.05(1.27-3.30)^{* *}$ & 0.003 \\
\hline $70 \leq$ age $<80$ & $4.24(2.68-6.70)^{* * *}$ & $<0.001$ & $2.46(1.53-3.94)^{* * * *}$ & $<0.001$ & $2.35(1.44-3.82)^{* *}$ & 0.001 \\
\hline $80 \leq$ age & $6.53(4.04-10.55)^{* * * *}$ & $<0.001$ & $2.98(1.80-4.91)^{* * * *}$ & $<0.001$ & $2.45(1.46-4.13)^{* *}$ & 0.001 \\
\hline Male $^{\mathrm{b}}$ & $1.19(1.01-1.41)^{*}$ & 0.037 & $1.24(1.05-1.47)^{*}$ & 0.012 & $1.22(1.03-1.45)^{*}$ & 0.020 \\
\hline \multicolumn{7}{|l|}{ Insurance premium (NT\$) } \\
\hline$\leq 22,800$ & 1 (reference) & & 1 (reference) & & 1 (reference) & \\
\hline$>22,800$ & $0.48(0.41-0.58)^{* * *}$ & $<0.001$ & $0.74(0.61-0.89)^{* *}$ & 0.002 & $0.80(0.66-0.96)^{*}$ & 0.019 \\
\hline \multicolumn{7}{|l|}{ Urbanization level } \\
\hline Urban & 1 (reference) & & 1 (reference) & & 1 (reference) & \\
\hline Suburban & $1.37(1.15-1.63)^{* * *}$ & $<0.001$ & $1.23(1.03-1.46)^{*}$ & 0.022 & $1.25(1.05-1.49)^{*}$ & 0.011 \\
\hline Rural & $1.18(0.89-1.55)$ & 0.250 & $0.96(0.72-1.26)$ & 0.753 & $0.97(0.74-1.29)$ & 0.851 \\
\hline \multicolumn{7}{|l|}{ Comorbidity } \\
\hline Dyslipidemia & $0.59(0.49-0.71)^{* *}$ & $<0.001$ & $0.84(0.67-1.04)$ & 0.113 & $0.86(0.69-1.07)$ & 0.178 \\
\hline Hypertension & $1.32(1.10-1.58)^{*}$ & 0.003 & $0.87(0.70-1.08)$ & 0.214 & $0.87(0.69-1.09)$ & 0.225 \\
\hline Diabetes Mellitus & $1.05(0.89-1.24)$ & 0.575 & $1.11(0.90-1.38)$ & 0.320 & $1.05(0.85-1.30)$ & 0.666 \\
\hline Obesity & $1.54(0.58-4.13)$ & 0.387 & $2.23(0.83-5.99)$ & 0.112 & $2.30(0.85-6.21)$ & 0.100 \\
\hline Chronic kidney disease & $2.06(1.50-2.84)^{* *}$ & $<0.001$ & $1.19(0.86-1.65)$ & 0.292 & $1.03(0.74-1.44)$ & 0.857 \\
\hline Chronic liver disease & $0.85(0.65-1.12)$ & 0.244 & $0.91(0.69-1.19)$ & 0.496 & $0.89(0.68-1.17)$ & 0.409 \\
\hline Arrhythmia $^{\mathrm{b}}$ & $2.93(2.43-3.52)^{* * *}$ & $<0.001$ & $1.50(1.22-1.85)^{* * * *}$ & $<0.001$ & $1.49(1.20-1.84)^{* * * *}$ & $<0.001$ \\
\hline Interstitial pulmonary diseases & $3.39(1.51-7.57)^{* *}$ & 0.003 & $2.15(0.96-4.84)$ & 0.063 & $1.99(0.88-4.47)$ & 0.098 \\
\hline Asthma $^{\mathrm{b}}$ & $2.00(1.68-2.39)^{* * *}$ & $<0.001$ & $1.65(1.38-1.98)^{* * * *}$ & $<0.001$ & $1.72\left(1.43-2.06^{* * *}\right.$ & $<0.001$ \\
\hline Malignant & $0.84(0.62-1.13)$ & 0.237 & $0.83(0.61-1.11)$ & 0.210 & $0.78(0.57-1.05)$ & 0.098 \\
\hline \multicolumn{7}{|l|}{ ASCVD } \\
\hline Coronary artery disease & $1.62(1.37-1.92)^{* * *}$ & $<0.001$ & $0.87(0.72-1.06)$ & 0.179 & $0.87(0.71-1.06)$ & 0.165 \\
\hline Peripheral vascular disease & $1.02(0.65-1.61)$ & 0.933 & $0.74(0.47-1.17)$ & 0.193 & $0.70(0.44-1.12)$ & 0.137 \\
\hline Ischemic stroke/TIA & $1.10(0.87-1.40)$ & 0.432 & $0.75(0.59-0.97)^{*}$ & 0.026 & $0.70(0.55-0.90)^{* *}$ & 0.006 \\
\hline Hemorrhagic stroke & $0.72(0.32-1.60)$ & 0.414 & $0.53(0.24-1.19)$ & 0.123 & $0.49(0.22-1.09)$ & 0.080 \\
\hline Heart failure $^{\mathrm{b}}$ & $5.32(4.45-6.37)^{* * *}$ & $<0.001$ & $2.17(1.74-2.71)^{* * * *}$ & $<0.001$ & $2.12(1.67-2.67)^{* * *}$ & $<0.001$ \\
\hline Left ventricular hypertrophy & $2.63(1.58-4.39)^{* * *}$ & $<0.001$ & $1.21(0.72-2.04)$ & 0.464 & $1.23(0.73-2.09)$ & 0.443 \\
\hline \multicolumn{7}{|l|}{ Co-medication } \\
\hline Digoxin $^{\mathrm{b}}$ & $5.06(4.10-6.25)^{* * *}$ & $<0.001$ & $1.50(1.17-1.93)^{* *}$ & 0.001 & $1.43(1.11-1.85)^{* *}$ & 0.006 \\
\hline Oral anticoagulant agents & $2.96(2.05-4.27)^{* * *}$ & $<0.001$ & $1.01(0.69-1.50)$ & 0.947 & $1.01(0.68-1.49)$ & 0.974 \\
\hline Oral antiplatelet agents & $1.69(1.44-1.99)^{* * *}$ & $<0.001$ & $1.12(0.92-1.36)$ & 0.275 & $1.12(0.91-1.36)$ & 0.285 \\
\hline Diuretics $^{\mathrm{b}}$ & $3.83(3.19-4.61)^{* * *}$ & $<0.001$ & $1.97(1.59-2.44)^{* * * *}$ & $<0.001$ & $1.94(1.57-2.39)^{* * *}$ & $<0.001$ \\
\hline CCB & $1.22(1.04-1.44)^{* * *}$ & $<0.001$ & $0.93(0.77-1.11)$ & 0.412 & $0.93(0.77-1.13)$ & 0.465 \\
\hline ACEI/ACB & $1.75(1.48-2.07)^{* * *}$ & $<0.001$ & $1.20(0.99-1.46)$ & 0.065 & $1.22(1.00-1.48)$ & 0.050 \\
\hline Beta blocker & $1.14(0.96-1.35)$ & 0.134 & $0.92(0.76-1.10)$ & 0.337 & $0.92(0.77-1.11)$ & 0.389 \\
\hline Metformin & $0.74(0.60-0.91)^{* *}$ & 0.004 & $0.72(0.55-0.93)^{*}$ & 0.011 & $0.73(0.57-0.94)^{*}$ & 0.015 \\
\hline Fibrate & $0.56(0.39-0.81)^{* *}$ & 0.002 & $0.81(0.55-1.18)$ & 0.275 & $0.82(0.56-1.20)$ & 0.313 \\
\hline \multicolumn{7}{|l|}{ COPD severity } \\
\hline \multicolumn{7}{|l|}{ Moderate exacerbations $^{b}$} \\
\hline 0 & 1 (reference) & & 1 (reference) & & 1 (reference) & \\
\hline 1 & $2.71(2.15-3.41)^{* * * *}$ & $<0.001$ & $1.57(1.23-2.01)^{* * * *}$ & $<0.001$ & $1.55(1.19-2.02)^{* *}$ & 0.001 \\
\hline$\geq 2$ & $2.77(2.25-3.42)^{* * *}$ & $<0.001$ & $1.49(1.17-1.89)^{* *}$ & 0.001 & $1.42(1.11-1.83)^{* *}$ & 0.006 \\
\hline \multicolumn{7}{|l|}{ Severe exacerbations $^{\mathbf{b}}$} \\
\hline 0 & 1 (reference) & & 1 (reference) & & 1 (reference) & \\
\hline 1 & $3.14(2.53-3.89)^{* * * *}$ & $<0.001$ & $1.57(1.23-1.99)^{* * * *}$ & $<0.001$ & $1.45(1.13-1.87)^{* *}$ & 0.004 \\
\hline$\geq 2$ & $4.84(3.64-6.43)^{* * * *}$ & $<0.001$ & $1.71(1.23-2.37)^{* *}$ & 0.001 & $1.33(0.96-1.85)$ & 0.089 \\
\hline
\end{tabular}

Table 2. Multivariate cox proportional hazard model analysis for variables related to the risk of $\mathrm{PH}$, stratified according to statins using ( ${ }^{a}$ Adjusted for age group, sex, income, comorbidity, co-medication, and COPD severity; ${ }^{b}$ Entry regression model after the stepwise multiple regression analysis; $\mathrm{COPD}=\mathrm{chronic}$ obstructive pulmonary disease; $\mathrm{PH}=$ pulmonary hypertension; $\mathrm{HR}=$ hazard ratio; $\mathrm{TWD}=$ Taiwan dollars; ASCVD $=$ atherosclerotic cardiovascular disease; TIA $=$ Transient ischemic attack; $C C B=$ calcium channel blocker; $\mathrm{ACEI}=$ angiotensin converting enzyme inhibitor; $\mathrm{ARB}=$ angiotensin receptor blocker; $*<0.05$; $* *<0.01 ; * * *<0.001)$. 


\begin{tabular}{|c|c|c|c|c|c|c|c|c|c|c|c|c|c|}
\hline \multirow[b]{2}{*}{ Kind of statins } & \multicolumn{4}{|c|}{ User $n=41163$} & \multicolumn{3}{|c|}{ Non-user $n=41163$} & \multirow{2}{*}{$\begin{array}{l}\text { Crude } \\
\text { HR }(95 \% \text { CI })\end{array}$} & \multirow[b]{2}{*}{$p$-value } & \multirow{2}{*}{\begin{tabular}{|l} 
Adjusted \\
HR $^{\mathrm{a}}(95 \% \mathrm{CI})$ \\
\end{tabular}} & \multirow[b]{2}{*}{$p$-value } & \multirow{2}{*}{\begin{tabular}{|l|} 
Subdistribution \\
HR $^{\mathrm{a}}(\mathbf{9 5} \% \mathrm{CI})$ \\
\end{tabular}} & \multirow[b]{2}{*}{$p$-value } \\
\hline & $\mathrm{N}$ & Events & Total of PY & Rate & Events & Total of PY & Rate & & & & & & \\
\hline simvastatin & 6575 & 42 & 28897.13 & 1.45 & 56 & 27522.95 & 2.03 & $0.72(0.48-1.07)$ & 0.102 & $0.66(0.44-1.00)$ & 0.050 & $0.71(0.47-1.06)$ & 0.094 \\
\hline lovastatin & 4385 & 37 & 19622.88 & 1.89 & 22 & 18513.47 & 1.19 & $1.60(0.94-2.71)$ & 0.082 & $1.63(0.95-2.79)$ & 0.076 & $1.64(0.97-2.77)$ & 0.063 \\
\hline pravastatin & 2588 & 13 & 10740.20 & 1.21 & 28 & 10820.43 & 2.59 & $0.47(0.24-0.90)^{*}$ & 0.023 & $0.44(0.23-0.86)^{*}$ & 0.016 & $0.45(0.23-0.89)^{*}$ & 0.021 \\
\hline $\begin{array}{l}\text { fluvastatin or } \\
\text { pitavastatin }\end{array}$ & 4084 & 19 & 15870.42 & 1.20 & 27 & 17050.70 & 1.58 & $0.76(0.42-1.36)$ & 0.351 & $0.74(0.41-1.35)$ & 0.331 & $0.78(0.43-1.39)$ & 0.391 \\
\hline atorvastatin & 16331 & 92 & 66581.49 & 1.38 & 148 & 67789.98 & 2.18 & $0.63(0.49-0.82)^{* *}$ & 0.001 & $0.63(0.23-0.82)^{* *}$ & 0.001 & $0.65(0.50-0.89)^{* *}$ & 0.001 \\
\hline rosuvastatin & 7200 & 39 & 28087.20 & 1.39 & 57 & 30232.80 & 1.89 & $0.74(0.49-1.11)$ & 0.142 & $0.75(0.49-1.13)$ & 0.167 & $0.77(0.51-1.17)$ & 0.227 \\
\hline
\end{tabular}

Table 3. Subgroup analysis of risk of $\mathrm{PH}$ in different kind of statins, stratified according to statins using

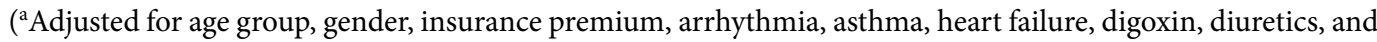
COPD severity; $\mathrm{HR}=$ hazard ratio; $\mathrm{PH}=$ pulmonary hypertension; $\mathrm{PY}=$ person-year; $\mathrm{Rate}=$ (event/personyear) $* 1000 ; *<0.05 ; * *<0.01 ; * *<0.001)$.

duration of statin use, this study completed a subgroup analysis of intensity, referring to the cDDD per month. The normal cDDD per month is 30 DDDs. However, the DDD is an average maintenance dose per day, so it may represent a moderate statin dose according to the drug's lipid-lowering effects ${ }^{11,24}$. Patients who used more than 20 DDDs of statin per month displayed a significantly lower risk of PH (sHR: 0.54, 95\% CI: 0.41-0.71). The exploration of the dose- and time-dependent effects of statins has been common in studies involving different populations, making the results more robust ${ }^{25-27}$.

The study is the first to explore the protective effects of statins against PH in COPD patients. In the study design, many important strengths can be observed. The Nation Health Insurance (NHI) database is one of the largest and most comprehensive medical population databases in the world, so the study had access to a larger sample size than other studies. Specifically, 41,163 COPD patients and 1,325 patients with PH related to COPD were included in the final analysis of this study. This is in comparison with other investigations, where the sample size of patients with $\mathrm{PH}$ related to COPD was only 40 to 60 patients ${ }^{28-33}$. This extensive sample size may provide enough power for statistical analysis. Another strength is the long observation time. The study relied on the database from 2002 to 2017 and included a five-year observation period, ensuring the availability of enough $\mathrm{PH}$ events for analysis. Further, the results were robust across several different definitions of statin drug exposure and observation duration.

In contrast, however, this study also had several limitations. The study population and outcomes were all defined based on the International Classification of Diseases, ninth revision, clinical modification (ICD-9-CM) or the International Classification of Diseases, 10th revision, clinical modification in the admissions record rather than on clinical diagnosis because the NHI database lacked examination results and laboratory data. Therefore, the definition of $\mathrm{PH}$ in this study was established according to a Chang et al. study that used the same NIH database. This previous study verified the accuracy of the diagnosis code (i.e., ICD-9-CM) by chart review in a medical centre hospital. The positive predictive value of using diagnosis codes in this regard was reported as up to $94.9 \%$. Then, the current study also used the second definition that combined the diagnosis codes and examinations to enhance the correct rate of $\mathrm{PH}$ diagnosis. Such examinations like right-heart catheterisation, echocardiographic, and chest X-ray were recommended by the 2015 European Society of Cardiology/European Respiratory Society guidelines for the diagnosis and treatment of pulmonary hypertension ${ }^{10}$. The limitations of the NHI database also include no access to smoking status, lifestyle, lung function or PH severity. The study also could not confirm that patients were taking treatments for $\mathrm{PH}$ or not. The reason for this is that patients in this population often do not fit the payment rules of $\mathrm{PH}$-specific therapy in the NHI programme. So, many used those drugs at their own expense, which would not be recorded in the NIH database.

In conclusion, statins may have a protective effect against $\mathrm{PH}$ through reducing the incidence of $\mathrm{PH}$ in patients with COPD. Moreover, the protective effect was dose- and time-dependent. An age of more than 60 years, male gender, low income, heart failure, arrhythmia, asthma and COPD severity were risk factors for PH. However, we could not identify a consistent benefit in protective effect between different kinds of statins. Further randomised controlled trials involving different statins and accurate statin exposure control are required.

\section{Methods}

Data sources. Taiwan established the NHI programme in 1995. The Taiwan NIH programme, which covers $99.6 \%$ of 23 million Taiwanese and $93 \%$ of hospitals, clinics and pharmacies, represents one of the largest and most comprehensive medical population databases in the world. We used the full population database with all its medical records and multiple cause of death datasets from the NHI programme from 2002 to $2017^{34}$. To maintain the privacy of patients, the identity numbers of patients, medical institutions and medical providers were encrypted through the application of anonymous codes. All researchers in Taiwan are required to follow the Computer-processed Personal Data Protection Law and should not attempt to decrypt and impair the privacy of patients. We independently conducted this study at a subcenter of the Health and Welfare Data Science Centers at Kaohsiung Medical University. This study was approved by the institutional review board of Kaohsiung Medical University Chung-Ho Memorial Hospital (KMUHIRB-EXEMPT(I)-20190032).

Study population. We identified newly diagnosed COPD patients from both outpatient and inpatient visit records from January 1, 2002 to December 31, 2015 (ICD-9-CM codes 490, 491, 492 and 496). Patients with more 


\begin{tabular}{|c|c|c|c|c|c|c|c|c|c|c|}
\hline \multirow[b]{2}{*}{ Group } & \multirow[b]{2}{*}{$\mathbf{N}$} & \multirow[b]{2}{*}{ Events } & \multirow[b]{2}{*}{ Total of PY } & \multirow[b]{2}{*}{ Rate } & \multirow{2}{*}{\begin{tabular}{|l|} 
Crude \\
HR $(95 \%$ CI $)$
\end{tabular}} & \multirow[b]{2}{*}{$p$-value } & \multirow{2}{*}{\begin{tabular}{|l|} 
Adjusted \\
HR $^{\mathrm{a}}(\mathbf{9 5} \% \mathrm{CI})$
\end{tabular}} & \multirow[b]{2}{*}{$p$-value } & \multirow{2}{*}{\begin{tabular}{|l|} 
Subdistribution \\
$\mathrm{HR}^{\mathrm{a}}(\mathbf{9 5} \% \mathrm{CI})$ \\
\end{tabular}} & \multirow[b]{2}{*}{$p$-value } \\
\hline & & & & & & & & & & \\
\hline \multicolumn{11}{|l|}{ cDDD } \\
\hline Non-user & 41163 & 338 & 171937.85 & 1.97 & 1 (reference) & & 1 (reference) & & 1 (reference) & \\
\hline cDDD $<28$ & 4992 & 61 & 19423.87 & 3.14 & $1.60(1.21-2.09)^{* *}$ & 0.001 & $1.36(1.03-1.79)^{*}$ & 0.031 & $1.36(1.02-1.82)^{*}$ & 0.038 \\
\hline $28 \leq \mathrm{cDDD}<90$ & 7147 & 59 & 26901.31 & 2.19 & $1.11(0.84-1.47)$ & 0.450 & $1.06(0.80-1.40)$ & 0.672 & $1.11(0.83-1.48)$ & 0.489 \\
\hline $90 \leq \mathrm{cDDD}<180$ & 6221 & 43 & 23988.18 & 1.79 & $0.91(0.66-1.25)$ & 0.560 & $0.89(0.65-1.23)$ & 0.479 & $0.94(0.67-1.30)$ & 0.695 \\
\hline $180 \leq \mathrm{cDDD}<365$ & 8472 & 40 & \begin{tabular}{|l|}
34531.87 \\
\end{tabular} & 1.16 & $0.59(0.42-0.82)^{* *}$ & 0.002 & $0.58(0.42-0.81)^{* *}$ & 0.001 & $0.66(0.47-0.92)^{*}$ & 0.015 \\
\hline $365 \leq \mathrm{cDDD}<730$ & 8495 & 25 & \begin{tabular}{|l|}
37496.93 \\
\end{tabular} & 0.67 & $0.34(0.23-0.51)^{* * *}$ & $<0.001$ & $0.37(0.24-0.55)^{* * * *}$ & $<0.001$ & $0.43(0.28-0.65)^{* * *}$ & $<0.001$ \\
\hline $730 \leq \mathrm{cDDD}$ & 5836 & 14 & 27464.22 & 0.51 & $0.26(0.15-0.44)^{* * *}$ & $<0.001$ & $0.26(0.15-0.45)^{* * * *}$ & $<0.001$ & $0.32(0.19-0.55)^{* * *}$ & $<0.001$ \\
\hline$p$ for trend test & $<0.001$ & & & & & & & & & \\
\hline \multicolumn{11}{|c|}{ Duration of statins use (year) } \\
\hline Non-user & 41163 & 338 & 171929.9123 & 1.97 & 1 (reference) & & 1 (reference) & & 1 (reference) & \\
\hline year $<0.5$ & 13448 & 125 & 51095.84931 & 2.45 & $1.24(1.01-1.52)^{*}$ & 0.039 & $1.15(0.93-1.41)$ & 0.203 & $1.12(0.91-1.38)$ & 0.301 \\
\hline $0.5 \leq$ year $<1$ & 7249 & 45 & 27638.78082 & 1.63 & $0.83(0.61-1.13)$ & 0.229 & $0.81(0.59-1.10)$ & 0.178 & $0.80(0.58-1.09)$ & 0.157 \\
\hline $1 \leq$ year $<2$ & 8931 & 32 & 37213.73973 & 0.86 & $0.44(0.30-0.63)^{* * *}$ & $<0.001$ & $0.44(0.31-0.64)^{* * * *}$ & $<0.001$ & $0.47(0.32-0.67)^{* * * *}$ & $<0.001$ \\
\hline $2 \leq$ year $<3$ & 5125 & 22 & 23182.96986 & 0.95 & $0.48(0.31-0.74)^{* *}$ & 0.001 & $0.51(0.33-0.79)^{* *}$ & 0.002 & $0.57(0.37-0.88)^{*}$ & 0.011 \\
\hline $3 \leq$ year & 6410 & 18 & 30677.67397 & 0.59 & $0.30(0.19-0.48)^{* * *}$ & $<0.001$ & $0.31(0.19-0.49)^{* * * *}$ & $<0.001$ & $0.35(0.22-0.56)^{* * * *}$ & $<0.001$ \\
\hline$p$ for trend test & $<0.001$ & & & & & & & & & \\
\hline \multicolumn{11}{|c|}{ Intensity (cDDD/month) } \\
\hline Non-user & 41163 & 338 & 171929.9123 & 1.97 & 1 (reference) & & 1 (reference) & & 1 (reference) & \\
\hline$<10$ & 4021 & 32 & 15812.85206 & 2.02 & $1.03(0.72-1.48)$ & 0.884 & $0.81(0.56-1.16)$ & 0.252 & $0.79(0.55-1.15)$ & 0.216 \\
\hline $10 \leq$ intensity $<20$ & 21619 & 149 & 91493.42192 & 1.63 & $0.83(0.68-1.01)$ & 0.056 & $0.83(0.69-1.01)$ & 0.064 & $0.87(0.71-1.05)$ & 0.142 \\
\hline $20 \leq$ & 15523 & 61 & 62502.73973 & 0.98 & $0.50(0.38-0.65)^{* * *}$ & $<0.001$ & $0.51(0.39-0.67)^{* * * *}$ & $<0.001$ & $0.54(0.41-0.71)^{* * * *}$ & $<0.001$ \\
\hline$p$ for trend test & $<0.001$ & & & & & & & & & \\
\hline
\end{tabular}

Table 4. Subgroup analysis of risk of $\mathrm{PH}$, stratified according to classification of cDDD, duration of statins use and intensity ( ${ }^{\mathrm{a}}$ Adjusted for age group, gender, insurance premium, arrhythmia, asthma, heart failure, digoxin, diuretics, and COPD severity.; $\mathrm{HR}=$ hazard ratio; $\mathrm{COPD}=$ chronic obstructive pulmonary disease; $\mathrm{PH}=$ pulmonary hypertension; $\mathrm{cDDD}=$ cumulative defined daily doses; $\mathrm{PY}=$ person-year; Rate $=($ event $/$ person-year) $* 1000 ; *<0.05 ; * *<0.01 ; * * *<0.001)$.

than one inpatient diagnosis or more than two consecutive outpatient diagnoses of COPD and who were treated using COPD medications according to outpatient claims for more than 28 days within one year after the primary COPD diagnosis date were defined as COPD patients. Patients with other etiologies of $\mathrm{PH}^{10}$ (Supplementary Table 3 ) and lung cancer (ICD-9-CM code 162) were excluded $(n=249,565)$. Patients who developed PH before their COPD diagnosis $(n=2,425)$ or died within 28 days after their COPD diagnosis $(n=15,148)$ were also excluded. The date of the primary diagnosis of COPD was defined as the index date.

Baseline characteristics and COPD severity. Baseline characteristics and COPD severity were confirmed by medical records in the one year after the index date according to ICD-9-CM codes or ICD-10-CM codes. The demographic data contained age, age group, gender, urbanisation level ${ }^{35,36}$ and insurance premium information. To adjust for other confounders that might influence the outcome, we listed comorbidities including a high risk of atherosclerotic cardiovascular disease (ASCVD) ${ }^{24}$ and other medications used in the treatment of PAH [e.g., digoxin, calcium channel blockers, warfarin]. Detailed baseline characteristics and definitions are shown in Supplementary Table 4. The assessment of exacerbation risk in the Global Initiative for Chronic Obstructive Lung Disease (GOLD) guidelines was used to define the severity of COPD. The category of severe exacerbations refers to exacerbations leading to emergency room or hospital admission, while moderate exacerbations were those not leading to hospital admission but where patients were treated with SABA plus antibiotics or oral corticosteroids ${ }^{37}$. Because the index date was the first COPD diagnosis date, the severity of COPD was confirmed at one year after the index date.

The baseline characteristics of the original cohort were used to perform PS matching by multivariate logistic regression (via OneToManyMTCH, a SAS procedure; SAS Institute, Cary, NC, USA). Data on age group, gender, insurance premium, urbanisation, hypertension, diabetes, heart failure, left ventricular hypertrophy and COPD severity were used to calculate the propensity score. We adopted a 1:1 matching scheme to generate the statin user and nonuser groups for analysis after follow-up ${ }^{38}$.

Exposure and outcomes assessment. For the statin exposure definition, to solve the issue of reverse causality, we established a six-month drug washout period according to the pharmacokinetic and pharmacodynamic properties of statins ${ }^{26,27}$. Patients with any level of statin exposure during the washout period were excluded. Patients needed to use at least one statin in the one year after COPD diagnosis to be defined as statin users. Conversely, patients who never received prescriptions for statins in the one year after COPD diagnosis were defined as statin nonusers. 


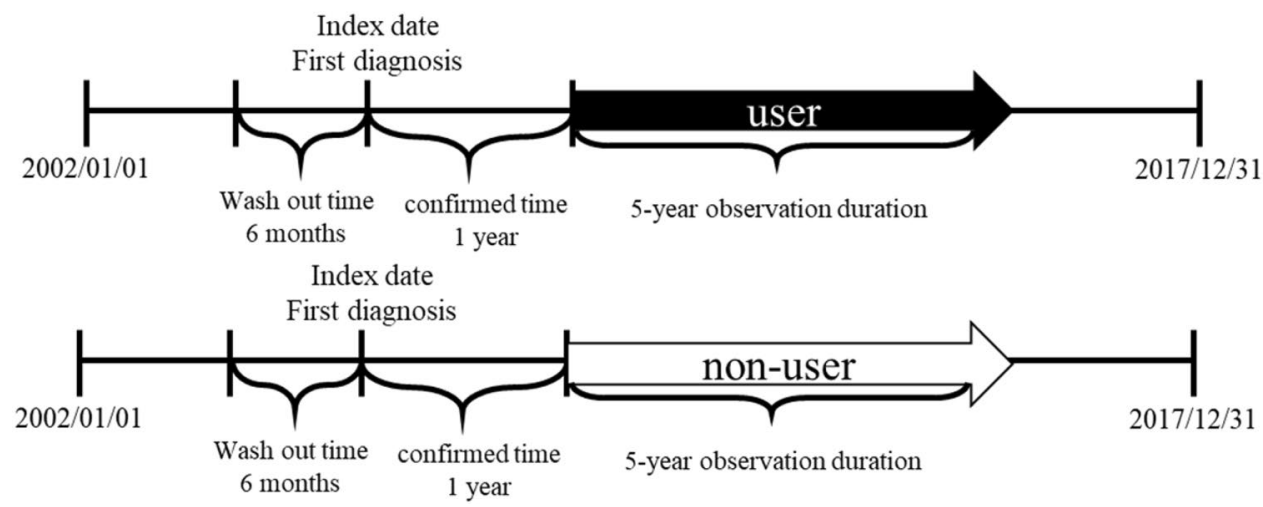

Figure 2. We set a five-year observation duration to reduce the influence of switching to different statins or discounting taking statins. The follow-up start form one year after index date and to the five years after index date.

The incidence of $\mathrm{PH}$ was the primary outcome in the study. To identify the event of $\mathrm{PH}$, we used the diagnosis code (i.e., 416.0, 416.8, or 416.9 in ICD-9-CM; I270, I272, I278, or I279 in ICD-10-CM) in the medical records to define the event of $\mathrm{PH}$. Then, we applied two criteria to confirm patients truly had $\mathrm{PH}$. For the first one, patients were required to have more than one inpatient diagnosis or emergency room admission for $\mathrm{PH}$ or more than two consecutive outpatient diagnoses of $\mathrm{PH}$ in one year. For the second, in the sensitivity analysis, patients had to undergo diagnostic examinations for $\mathrm{PH}$ (e.g., right-heart catheterisation, echocardiography, or chest X-ray) and receive a diagnosis of $\mathrm{PH}$ at the same time. We used the first criterion in the main analysis ${ }^{4,7}$.

To assess the influence of incidence among different kinds of statins, doses and time frames, this study included subgroup analyses. There were seven statins that were prescribed in Taiwan during the study period. The patients' statin group was determined based on their most-used statin during the five-year observation period. We analysed the dose-dependent effect by considering the DDD, which was established by the World Health Organization to standardise the doses of different kinds of statins. The cDDD was the total amount of statin exposure during the five-year observation period and was divided into six levels (i.e., $\leq 28,28-90,90-180,180-365$, $365-730$, and $\geq 730 \mathrm{cDDD}$ ). Additionally, the duration of statin use was calculated by year and divided into five levels (i.e., $\leq 0.5,0.5-1,1-2,2-3$, and $\geq 3$ years). The intensity was calculated by dividing the cDDD by the duration of statin use during the whole five-year observation period. Then, we divided such into three levels to compare weather the intensity could affect the protective effect of statins ${ }^{39}$ (i.e., $\leq 10,10-20$, and $\geq 20 \mathrm{cDDD} / \mathrm{month}$ ).

Follow-up time. The five-year follow-up period began at one year after the index date. Users and nonusers alike stopped participating at the occurrence of $\mathrm{PH}$ or censoring. If patients died before the occurrence of $\mathrm{PH}$ or were not diagnosed with $\mathrm{PH}$ by the end of the observation period, they were defined as being censors. The definition of censors was the same in both the user and nonuser groups. During the observation period, any statin exposure would not change the user group (Fig. 2).

Statistical analysis. For baseline characteristics, continuous variables were presented as means (standard deviations) and categorical variables were presented as percentages. Continuous variables were analysed by Student's $\mathrm{t}$-test or analysis of variance and categorical variables were analysed by Fisher's exact test or chi-squared test if the value was less than 30 . The crude incidence of $\mathrm{PH}$ was estimated as the total number of events during the five-year observation period divided by total person-years. This study conducted a CPH model to estimate the HR between the two user groups. The multivariate model was adjusted according to demographic characteristics, gender, comorbidities, comedication use and COPD severity to calculate the aHR. To reduce the influence of interaction and collinear effects between characteristics, stepwise selection was performed to select important factors to construct the multivariate regression model for subgroup analysis. Because of the competing risk of death, the Fine and Gray competing risk model was used to estimate the sHR in the primary outcome ${ }^{40}$.

All of the above analyses were performed using the SAS version 9.4. software program (SAS Institute, Cary, NC, USA). Statistical significance was determined as two-tailed and $\alpha=0.05$.

Received: 5 September 2019; Accepted: 4 February 2020;

Published online: 20 February 2020

\section{References}

1. Hsu, C. H. et al. 2014 Guidelines of Taiwan Society of Cardiology (TSOC) for the Management of Pulmonary Arterial Hypertension. Acta Cardiol. Sin. 30, 401-444 (2014).

2. Simonneau, G. et al. Haemodynamic definitions and updated clinical classification of pulmonary hypertension. Eur Respir J 53, https://doi.org/10.1183/13993003.01913-2018 (2019).

3. Badesch, D. B. et al. Pulmonary arterial hypertension: baseline characteristics from the REVEAL Registry. Chest 137, 376-387, https://doi.org/10.1378/chest.09-1140 (2010).

4. Peacock, A. J., Murphy, N. F., McMurray, J. J., Caballero, L. \& Stewart, S. An epidemiological study of pulmonary arterial hypertension. Eur. Respir. J. 30, 104-109, https://doi.org/10.1183/09031936.00092306 (2007).

5. McGoon, M. D. et al. Pulmonary arterial hypertension: epidemiology and registries. J. Am. Coll. Cardiol. 62, D51-59, https://doi. org/10.1016/j.jacc.2013.10.023 (2013). 
6. Ling, Y. et al. Changing demographics, epidemiology, and survival of incident pulmonary arterial hypertension: results from the pulmonary hypertension registry of the United Kingdom and Ireland. Am. J. Respir. Crit. Care Med. 186, 790-796, https://doi. org/10.1164/rccm.201203-0383OC (2012).

7. Chang, W. T. et al. Prognostic Factors in Patients With Pulmonary Hypertension-A Nationwide Cohort Study. J Am Heart Assoc 5, https://doi.org/10.1161/JAHA.116.003579 (2016).

8. Hurdman, J. et al. ASPIRE registry: assessing the Spectrum of Pulmonary hypertension Identified at a REferral centre. Eur. Respir. J. 39, 945-955, https://doi.org/10.1183/09031936.00078411 (2012).

9. Cuttica, M. J. Pulmonary hypertension associated with lung diseases and hypoxemia. Heart Fail. Rev. 21, 299-308, https://doi. org/10.1007/s10741-016-9551-x (2016).

10. Galie, N. et al. 2015 ESC/ERS Guidelines for the diagnosis and treatment of pulmonary hypertension: The Joint Task Force for the Diagnosis and Treatment of Pulmonary Hypertension of the European Society of Cardiology (ESC) and the European Respiratory Society (ERS): Endorsed by: Association for European Paediatric and Congenital Cardiology (AEPC), International Society for Heart and Lung Transplantation (ISHLT). Eur. Heart J. 37, 67-119, https://doi.org/10.1093/eurheartj/ehv317 (2016).

11. Wierzbicki, A. S., Poston, R. \& Ferro, A. The lipid and non-lipid effects of statins. Pharmacology Therapeutics 99, 95-112, https://doi. org/10.1016/s0163-7258(03)00055-x (2003).

12. So, J. Y., Dhungana, S., Beros, J. J. \& Criner, G. J. Statins in the treatment of COPD and asthma-where do we stand? Curr. Opin. Pharmacol. 40, 26-33, https://doi.org/10.1016/j.coph.2018.01.001 (2018).

13. Mroz, R. M. et al. Anti-inflammatory effects of atorvastatin treatment in chronic obstructive pulmonary disease. A controlled pilot study. J. Physiol. Pharmacol. 66, 111-128 (2015).

14. Maneechotesuwan, K., Wongkajornsilp, A., Adcock, I. M. \& Barnes, P. J. Simvastatin Suppresses Airway IL-17 and Upregulates IL-10 in Patients With Stable COPD. Chest 148, 1164-1176, https://doi.org/10.1378/chest.14-3138 (2015).

15. Sun, X. \& Ku, D. D. Rosuvastatin provides pleiotropic protection against pulmonary hypertension, right ventricular hypertrophy, and coronary endothelial dysfunction in rats. Am. J. Physiol. Heart Circ. Physiol 294, H801-809, https://doi.org/10.1152/ ajpheart.01112.2007 (2008).

16. Nishimura, T. et al. Simvastatin attenuates smooth muscle neointimal proliferation and pulmonary hypertension in rats. Am. J. Respir. Crit. Care Med. 166, 1403-1408, https://doi.org/10.1164/rccm.200203-268OC (2002).

17. Seeger, H., Mueck, A. O. \& Lippert, T. H. Fluvastatin increases prostacyclin and decreases endothelin production by human umbilical vein endothelial cells. Int. J. Clin. Pharmacol. Ther. 38, 270-272, https://doi.org/10.5414/cpp38270 (2000).

18. Ikeda, T. et al. Inhibitory effects of simvastatin on platelet-derived growth factor signaling in pulmonary artery smooth muscle cells from patients with idiopathic pulmonary arterial hypertension. J. Cardiovasc. Pharmacol. 55, 39-48, https://doi.org/10.1097/ FJC.0b013e3181c0419c (2010).

19. Murata, T. et al. Statin protects endothelial nitric oxide synthase activity in hypoxia-induced pulmonary hypertension. Arterioscler. Thromb. Vasc. Biol. 25, 2335-2342, https://doi.org/10.1161/01.ATV.0000186184.33537.48 (2005).

20. Xing, X. Q. et al. Statins may ameliorate pulmonary hypertension via RhoA/Rho-kinase signaling pathway. Med. Hypotheses 68, 1108-1113, https://doi.org/10.1016/j.mehy.2006.09.034 (2007).

21. Reed, R. M. et al. Statin therapy is associated with decreased pulmonary vascular pressures in severe COPD. COPD 8, 96-102, https://doi.org/10.3109/15412555.2011.558545 (2011).

22. Pugh, M. E. et al. Causes of pulmonary hypertension in the elderly. Chest 146, 159-166, https://doi.org/10.1378/chest.13-1900 (2014).

23. Abdel-Qadir, H. et al. Importance of Considering Competing Risks in Time-to-Event Analyses: Application to Stroke Risk in a Retrospective Cohort Study of Elderly Patients With Atrial Fibrillation. Circ. Cardiovasc. Qual. Outcomes 11, e004580, https://doi. org/10.1161/CIRCOUTCOMES.118.004580 (2018).

24. Grundy, S. M. et al. 2018 AHA/ACC/AACVPR/AAPA/ABC/ACPM/ADA/AGS/APhA/ASPC/NLA/PCNA Guideline on the Management of Blood Cholesterol. Circulation, CIR0000000000000625, https://doi.org/10.1161/CIR.0000000000000625 (2018).

25. Langballe, R. et al. Statin use and risk of contralateral breast cancer: a nationwide cohort study. Br. J. Cancer 119, 1297-1305, https:// doi.org/10.1038/s41416-018-0252-1 (2018).

26. Liu, J. C. et al. Statins dose-dependently exert a chemopreventive effect against lung cancer in COPD patients: a population-based cohort study. Oncotarget 7, 59618-59629, https://doi.org/10.18632/oncotarget.11162 (2016).

27. Liu, J. C. et al. Statins dose-dependently exert a significant chemopreventive effect on colon cancer in patients with chronic obstructive pulmonary disease: A population-based cohort study. Oncotarget 7, 65270-65283, https://doi.org/10.18632/ oncotarget.11263 (2016).

28. Arian, A., Moghadam, S. G., Kazemi, T. \& Hajihosseini, M. The Effects of Statins on Pulmonary Artery Pressure in Patients with Chronic Obstructive Pulmonary Disease: A Randomized Controlled Trial. J. Res. Pharm. Pract. 6, 27-30, https://doi. org/10.4103/2279-042X.200985 (2017).

29. Chogtu, B. et al. A prospective, randomized study: Evaluation of the effect of rosuvastatin in patients with chronic obstructive pulmonary disease and pulmonary hypertension. Indian. J. Pharmacol. 48, 503-508, https://doi.org/10.4103/0253-7613.190721 (2016).

30. Moosavi, S. A. J., Raji, H., Faghankhani, M., Yazdani, R. \& Esmaeili, M. Evaluation of the Effects of Atorvastatin on the Treatment of Secondary Pulmonary Hypertension due to Chronic Obstructive Pulmonary Diseases: A Randomized Controlled. Trial. Iran. Red. Crescent Me 15, 649-654, https://doi.org/10.5812/ircmj.8267 (2013).

31. Zeng, W. J. et al. Atorvastatin in pulmonary arterial hypertension (APATH) study. Eur. Respir. J. 40, 67-74, https://doi. org/10.1183/09031936.00149011 (2012).

32. Wilkins, M. R. et al. Simvastatin as a treatment for pulmonary hypertension trial. Am. J. Respir. Crit. Care Med. 181, 1106-1113, https://doi.org/10.1164/rccm.2009111-6990C (2010).

33. Lee, T. M., Chen, C. C., Shen, H. N. \& Chang, N. C. Effects of pravastatin on functional capacity in patients with chronic obstructive pulmonary disease and pulmonary hypertension. Clin. Sci. 116, 497-505, https://doi.org/10.1042/CS20080241 (2009).

34. Hsieh, C.-Y. et al. Taiwan's National Health Insurance Research Database: past and future. Clin. Epidemiol. 11, 349-358, https://doi. org/10.2147/clep.s196293 (2019).

35. Cheng, B. R. et al. Rural-urban disparities in family physician practice patterns: A nationwide survey in Taiwan. Int. J. Health Plann Manage. https://doi.org/10.1002/hpm.2662 (2018).

36. Liu, C. Y. et al. development stratification of Taiwan townships into sampling design of large scale health interview survey. J. Health Manag. 4, 1-22 (2006).

37. Wang, M. T. et al. Association of Cardiovascular Risk With Inhaled Long-Acting Bronchodilators in Patients With Chronic Obstructive Pulmonary Disease: A Nested Case-Control Study. JAMA Intern. Med. 178, 229-238, https://doi.org/10.1001/ jamainternmed.2017.7720 (2018)

38. S., L. in Proceedings of the 29th SAS Users Group International Conference (Canada, 2004).

39. Chang, Y. T. et al. Dose-Dependent Relationship Between Metformin and Colorectal Cancer Occurrence Among Patients with Type 2 Diabetes-A Nationwide Cohort Study. Transl. Oncol. 11, 535-541, https://doi.org/10.1016/j.tranon.2018.02.012 (2018).

40. Austin, P. C., Lee, D. S. \& Fine, J. P. Introduction to the Analysis of Survival Data in the Presence of Competing Risks. Circulation 133, 601-609, https://doi.org/10.1161/CIRCULATIONAHA.115.017719 (2016). 


\section{Acknowledgements}

This study was based in part on data from the NHIRD provided by the Bureau of National Health Insurance (BNHI) of the Ministry of Health and Welfare. The conclusions presented in this study are those of the authors and do not necessarily reflect the views of the BNHI, the the Ministry of Health and Welfare. This work was supported by the Kaohsiung Medical University (KMU-Q106011). We are grateful to Kaohsiung Medical University for providing administrative and funding support.

\section{Author contributions}

All authors conceived the study design; Wen-Ting conducted the experiment and wrote the manuscripts. All authors reviewed the manuscript.

\section{Competing interests}

The authors declare no competing interests.

\section{Additional information}

Supplementary information is available for this paper at https://doi.org/10.1038/s41598-020-59828-0.

Correspondence and requests for materials should be addressed to C.-Y.C.

Reprints and permissions information is available at www.nature.com/reprints.

Publisher's note Springer Nature remains neutral with regard to jurisdictional claims in published maps and institutional affiliations.

Open Access This article is licensed under a Creative Commons Attribution 4.0 International License, which permits use, sharing, adaptation, distribution and reproduction in any medium or format, as long as you give appropriate credit to the original author(s) and the source, provide a link to the Creative Commons license, and indicate if changes were made. The images or other third party material in this article are included in the article's Creative Commons license, unless indicated otherwise in a credit line to the material. If material is not included in the article's Creative Commons license and your intended use is not permitted by statutory regulation or exceeds the permitted use, you will need to obtain permission directly from the copyright holder. To view a copy of this license, visit http://creativecommons.org/licenses/by/4.0/.

(C) The Author(s) 2020 